\title{
LiBeB Evolution: Three Models
}

\author{
Reuven Ramaty \\ NASA/GSFC, Greenbelt, MD 20771, USA \\ Richard E. Lingenfelter \\ CASS/UCSD, LaJolla, CA 92093, USA \\ Benzion Kozlovsky \\ Tel Aviv University, Israel
}

\begin{abstract}
.
We consider the three principal $\mathrm{LiBeB}$ evolutionary models, $\mathrm{CRI}$ in which the cosmic-ray source at all epochs of Galactic evolution is the average ISM, CRI+LECR in which metal enriched low energy cosmic rays (LECRs) are superimposed onto the CRI cosmic rays, and CRS in which the cosmic-ray source, accelerated in superbubbles, is constant, independent of the ISM metallicity. By considering the evolutionary trend of $\log (\mathrm{Be} / \mathrm{H})$ vs. both $[\mathrm{Fe} / \mathrm{H}]$ and $[\mathrm{O} / \mathrm{H}]$, we demonstrate that the CRI model is energetically untenable. We present evolutionary trends for ${ }^{11} \mathrm{~B} /{ }^{10} \mathrm{~B}$ and $\mathrm{B} / \mathrm{Be}$ which, combined with future precision measurements, could distinguish between the CRS and CRI+LECR models. We show that delayed LiBeB synthesis in the CRS model, due to the transport of the cosmic rays, could explain why $\log (\mathrm{Be} / \mathrm{H})$ is steeper vs. $[\mathrm{O} / \mathrm{H}]$ than vs. $[\mathrm{Fe} / \mathrm{H}]$. We also show that delayed deposition of $\mathrm{Fe}$ into star forming regions, due to its incorporation into high velocity dust, could provide an explanation for the possible rise of $[\mathrm{O} / \mathrm{Fe}]$ with decreasing $[\mathrm{Fe} / \mathrm{H}]$. Observations of refractory and volatile $\alpha$-elements could test this scenario. There seems to be a need for pregalactic or extragalactic ${ }^{6} \mathrm{Li}$ sources.
\end{abstract}

\section{Introduction}

Cosmic-ray driven nucleosynthesis has been known to be important for the origin of the light elements $\mathrm{Li}, \mathrm{Be}$ and $\mathrm{B}(\mathrm{LiBeB})$ for three decades (Reeves, Fowler, \& Hoyle 1970). But constraints on the relevant evolutionary models could only be obtained after $\mathrm{LiBeB}$ abundances of low metallicity stars started to become available (e.g. Ryan et al. 1990). The principal three models currently considered are: (i) the cosmic-ray interstellar model (hereafter CRI), in which the cosmic-ray source composition at all epochs of Galactic evolution is assumed to be similar to that of the average ISM at that epoch (Vangioni-Flam et al. 1990; Fields \& Olive 1999); (ii) the CRI+LECR model, in which metal enriched low energy cosmic rays (LECRs) are superimposed onto the CRI cosmic rays 
(Cassé, Lehoucq, \& Vangioni-Flam 1995; Vangioni-Flam et al. 1996; Ramaty, Kozlovsky, \& Lingenfelter 1996); and (iii) the cosmic-ray superbubble model (hereafter CRS), in which the cosmic-ray source composition is taken to be constant, independent of the ISM metallicity (Ramaty et al. 1997;2000; Higdon, Lingenfelter, Ramaty 1998). Both the CRS cosmic rays and the LECRs are thought to be accelerated out of supernova enriched matter in superbubbles.

Because of the excess of the observed Be abundances in low metallicity stars over the predictions of the CRI model, and motivated by reports of the detection of $\mathrm{C}$ and $\mathrm{O}$ nuclear gamma-ray lines from the Orion star formation region (Bloemen et al. 1994), LECRs, enriched in $\mathrm{C}$ and $\mathrm{O}$ relative to protons and $\alpha$ particles, were superimposed on the CRI cosmic rays, hence the CRI+LECR model . These LECRs, with maximum energies not exceeding about $100 \mathrm{MeV} /$ nucleon, were thought (e.g. Ramaty 1996) to be responsible for the gamma rays reported from Orion. It was suggested that such enriched LECRs might be accelerated out of metal-rich winds of massive stars and supernova ejecta (Bykov \& Bloemen 1994; Ramaty et al. 1996; Parizot, Cassé, \& Vangioni-Flam 1997) by an ensemble of shocks in superbubbles (Bykov \& Fleishman 1992; Parizot Cassé, \& Vangioni-Flam 1997). The Orion gamma-ray data, however, have been retracted (Bloemen et al. 1999). Nonetheless, as the possible existence of the postulated LECRs remains, new gamma-ray line data are needed to determine the role of LECRs in LiBeB production.

Recent $\mathrm{O}$ abundance data, which suggest that $[\mathrm{O} / \mathrm{Fe}]$ increases with decreasing $[\mathrm{Fe} / \mathrm{H}]$ at low metallicities (Israelian et al. 1998; Boesgaard et al. 1999), led Fields and Olive (1999) to reexamine the viability of the CRI model. More recent measurements (Fulbright \& Kraft 1999; Westin et al. 1999) argue against such an $[\mathrm{O} / \mathrm{Fe}]$ increase. But as demonstrated in (Ramaty et al. 2000), and also shown below, this model is inconsistent with cosmic-ray energetics, an $[\mathrm{O} / \mathrm{Fe}]$ increase notwithstanding.

Alternatively, it was suggested (Lingenfelter, Ramaty, \& Kozlovsky 1998; Higdon et al. 1998), that the Be evolution can be best understood in the CRS model, in which the cosmic-ray metals at all epochs of Galactic evolution are accelerated predominantly out of supernova ejecta. Lingenfelter et al. (1998) and Lingenfelter \& Ramaty (1999) showed that the arguments (e.g. Meyer, Drury, \& Ellison 1997) against the supernova ejecta origin of the current epoch cosmic rays can be answered, and Higdon et al. (1998) and Higdon, Lingenfelter, \& Ramaty (1999) showed that the most likely scenario is collective acceleration by successive supernova shocks of ejecta-enriched matter in the interiors of superbubbles. This scenario is consistent with the delay between nucleosynthesis and acceleration (time scales $\sim 10^{5} \mathrm{yr}$ ), suggested by the ${ }^{59} \mathrm{Co}$ and ${ }^{59} \mathrm{Ni}$ observations (Wiedenbeck et al. 1999). In both the CRS and CRI+LECR models, the bulk of the $\mathrm{Be}$ in the early Galaxy is produced by accelerated $\mathrm{C}$ and $\mathrm{O}$ interacting with ambient $\mathrm{H}$ and $\mathrm{He}$. That these "inverse reactions" are dominant in the early Galaxy was first suggested by Duncan, Lambert, \& Lemke (1992).

In the present paper we present results from a complete set of $\mathrm{LiBeB}$ evolutionary calculations for all three models using our production code described in Ramaty et al. (1997) and evolutionary code detailed in Ramaty et al. (2000). 

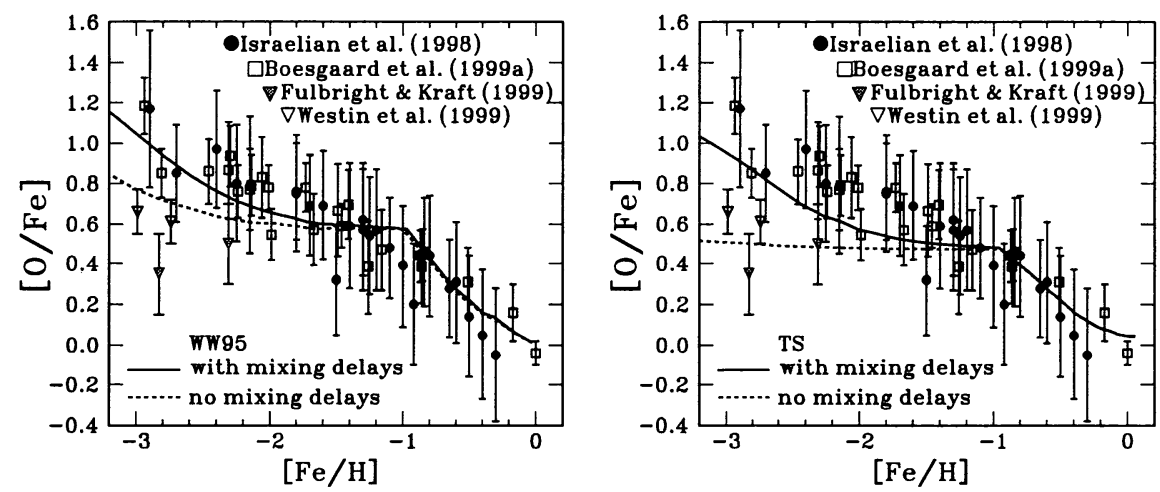

Figure 1. Galactic evolution of $[\mathrm{O} / \mathrm{Fe}]$ vs. $[\mathrm{Fe} / \mathrm{H}]$ for supernova $\mathrm{O}$ and $\mathrm{Fe}$ yields taken from minimum Fe yield models of Woosley \& Weaver (1995, WW95 shown in the left panel) and Tsujimoto \& Shigeyama (1998, TS shown in the right panel), assuming zero and finite $\mathrm{Fe}$ and $\mathrm{O}$ mixing delay times, $\tau_{\mathrm{Fe}}(\mathrm{mix})=30 \mathrm{Myr}$ and $\tau_{\mathrm{O}}(\mathrm{mix})$ $=1 \mathrm{Myr}$, and including contributions from Type Ia supernovae. The characteristic halo and disk infall times are $10 \mathrm{Myr}$ and $5 \mathrm{Gyr}$, respectively, the star formation rate coefficient is $0.5 \mathrm{Gyr}^{-1}$, and the ratio of halo-to-disk masses is 0.1. (See Ramaty et al. 2000 for more details).

\section{Analysis}

Figure 1 shows the evolution of $[\mathrm{O} / \mathrm{Fe}]$ as a function of $[\mathrm{Fe} / \mathrm{H}]$. In order to account for the possible rise of $[\mathrm{O} / \mathrm{Fe}]$ with decreasing $[\mathrm{Fe} / \mathrm{H}]$, we introduced mixing delays, i. e. the delayed deposition of the synthesized products into the star forming regions due to differences in transport and mixing. We choose a short mixing time for oxygen because we expect the bulk of the $\mathrm{O}$ and other volatiles in the ejecta to mix with the ISM after the remnant slows down to local sound speeds. But we consider longer mixing times for $\mathrm{Fe}$, assuming that the bulk of the ejected $\mathrm{Fe}$ is incorporated into high velocity refractory dust grains which continue moving for longer periods of time before they stop and can be incorporated into newly forming stars. The incorporation of a large fraction of the synthesized $\mathrm{Fe}$ into dust grains is supported by observations of both supernova $1987 \mathrm{~A}$ and the Galactic $1.809 \mathrm{MeV}$ gamma-ray line resulting from the decay of ${ }^{26} \mathrm{Al}$ (for more details and references see Ramaty et al. 2000). We see that with the mixing delays (solid curves in Figure 1) both the WW95 and TS cases (see figure caption) become consistent with the Israelian et al. (1998) and Boesgaard et al. (1999a) data, showing that delayed Fe deposition could indeed be the cause for the rise of $[\mathrm{O} / \mathrm{Fe}]$. In this connection, it is interesting to note that, unlike $[\mathrm{O} / \mathrm{Fe}]$, the abundance ratios of the $\alpha$-nuclei $\mathrm{Mg}, \mathrm{Si}, \mathrm{Ca}$ and $\mathrm{Ti}$ relative to $\mathrm{Fe}$ do not increase with decreasing $[\mathrm{Fe} / \mathrm{H}]$ below $[\mathrm{Fe} / \mathrm{H}]=-1$ (Ryan, Norris, \& Beers 1996). This may be consistent with the fact that these elements are also refractory, and thus are affected by mixing in the same way as is Fe. A test may be provided by sulfur, which is volatile, and thus should show 

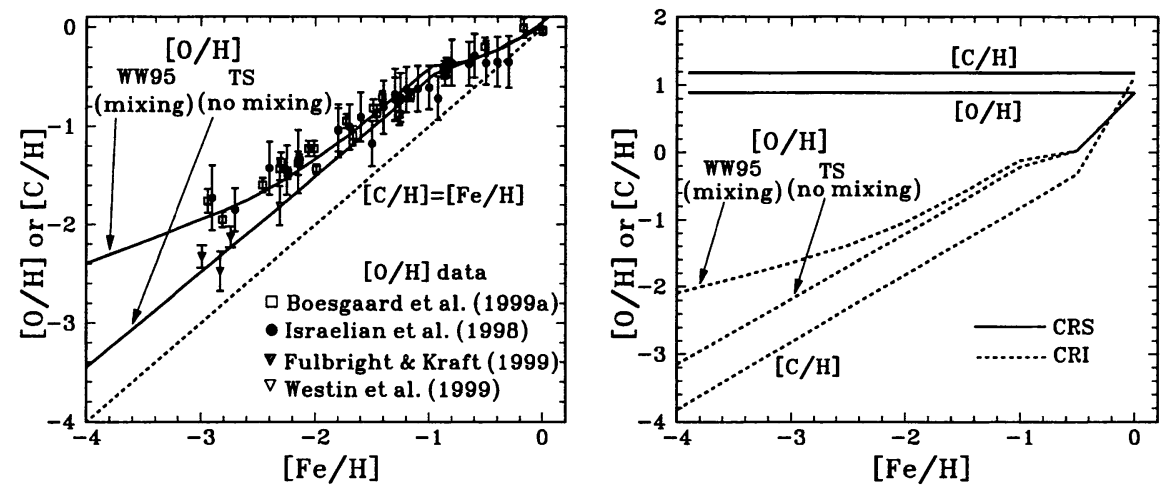

Figure 2. The employed ISM (left panel) and cosmic-ray source (right panel) $\mathrm{C}$ and $\mathrm{O}$ abundances as functions of $[\mathrm{Fe} / \mathrm{H}]$. For the ISM, $[\mathrm{O} / \mathrm{H}]$ is obtained from the evolutionary calculation for the two limiting cases, WW95 with mixing delays and TS with no mixing delays (Figure 1$).[\mathrm{C} / \mathrm{Fe}]$ is taken constant. For the cosmic rays the abundances are normalized to the solar values, i.e. $[\mathrm{X} / \mathrm{Fe}] \equiv \log (\mathrm{X} / \mathrm{Fe})-\log (\mathrm{X} / \mathrm{Fe})_{\odot}$ where $\mathrm{X}$ stands for $\mathrm{C}$ or $\mathrm{O}$. For the $\mathrm{CRS} \operatorname{model}[\mathrm{C} / \mathrm{H}]$ and $[\mathrm{O} / \mathrm{H}]$ are independent of $[\mathrm{Fe} / \mathrm{H}]$. For the $\mathrm{CRI}$ model, $[\mathrm{C} / \mathrm{H}]$ and $[\mathrm{O} / \mathrm{H}]$ are based on the ISM values with enhancement factors consistent with shock acceleration theory (see text), except at $[\mathrm{Fe} / \mathrm{H}]=0$ where the cosmic ray CRS and CRI values are equal.

a rise similar to the rise of $[\mathrm{O} / \mathrm{Fe}]$ vs. decreasing $[\mathrm{Fe} / \mathrm{H}]$ (G. Israelian, private communication, 1999).

Figure 2 shows the employed $\mathrm{C}$ and $\mathrm{O}$ abundances of the ISM and the cosmic ray source. For the ISM (left panel) we take $[\mathrm{C} / \mathrm{H}] \simeq[\mathrm{Fe} / \mathrm{H}]$, because at early times both the $\mathrm{C}$ and $\mathrm{Fe}$ come primarily from core collapse supernovae of massive stars, while at later times the increased $\mathrm{C}$ contribution from the winds of intermediate mass stars is compensated by the $\mathrm{Fe}$ contribution from the thermonuclear supernovae of the white dwarf remnants of such stars (see Timmes, Woosley \& Weaver 1995). The $\mathrm{O}$ abundances follows from the results of evolutionary calculations shown in Figure 1. Unlike in Ramaty et al. (2000), where we used a constant He abundances, here we allow $\mathrm{He} / \mathrm{H}$ (by number) to vary slowly from 0.08 at very low metallicities to 0.1 at $[\mathrm{Fe} / \mathrm{H}]=0$. For the cosmicray source (right panel), we define the logarithmic ratios $[\mathrm{C} / \mathrm{H}]$ and $[\mathrm{O} / \mathrm{H}]$ in the same way as is done for the corresponding ISM values, including normalization to solar (not current epoch cosmic ray source) abundances. As the CRS cosmic rays are accelerated primarily out of supernova ejecta enriched superbubbles, $[\mathrm{C} / \mathrm{H}]$ and $[\mathrm{O} / \mathrm{H}]$ are constant, set equal to current epoch cosmic-ray values. For the $\mathrm{CRI}$ model we scale $[\mathrm{C} / \mathrm{H}]$ and $[\mathrm{O} / \mathrm{H}]$ to the ISM values with enhancement factors of 1.5 and 2, consistent with the mass-to-charge dependent acceleration of volatiles (Ellison, Drury \& Meyer 1997), except at $[\mathrm{Fe} / \mathrm{H}]=0$ where the cosmic ray CRS and CRI values are equal. For details on the rest of the employed cosmic-ray sources abundances see Ramaty et al. (2000). For the LECRs we adopt the CRS abundances. The CRS, CRI and LECR source energy spectra are 

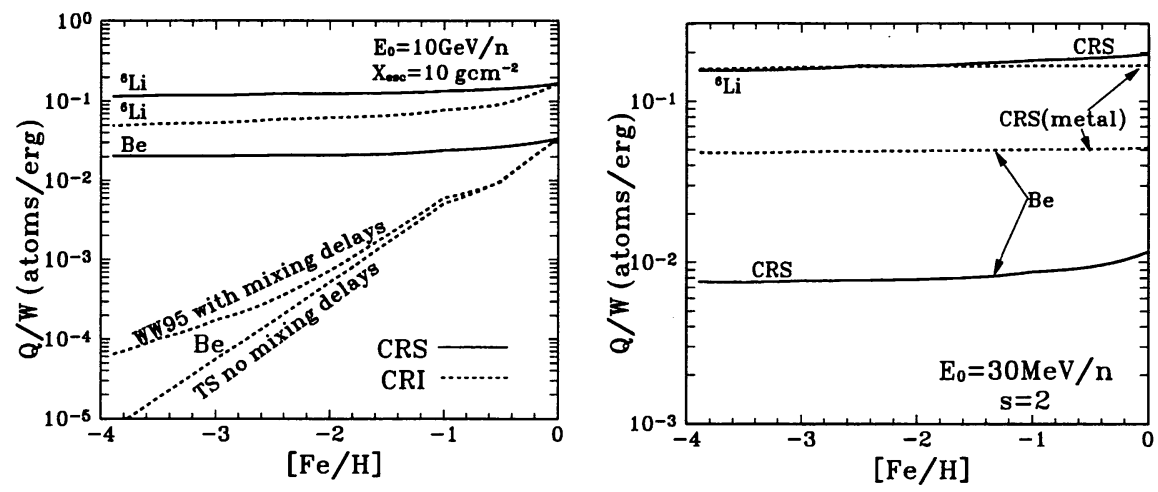

Figure 3. Number of $\mathrm{Be}$ and ${ }^{6} \mathrm{Li}$ atoms produced per unit cosmic-ray energy. Left panel - for the CRS and CRI models using a cosmic-ray source momentum power law spectral index of 2.5. For the CRI Be the two limiting mixing cases are shown. For ${ }^{6} \mathrm{Li}$ in both the CRS and CRI models, and for Be in the CRS model, the mixing cases do not affect $\mathrm{Q} / \mathrm{W}$. Right panel - for the LECR model with a turnover energy of 30 $\mathrm{MeV} /$ nucleon, spectral index of 2, CRS and CRS(metal) abundances, where the latter is identical to CRS, except that the proton and $\alpha$ particle abundances are set to zero. For these abundances the mixing cases have no effect.

power laws in momentum with high energy exponential cutoffs (characteristic energy $E_{0}$ ), which we set to an ultrarelativistic value for the CRS and CRI cosmic rays and to $30 \mathrm{MeV} /$ nucleon for the LECRs.

Figure 3 shows the resultant $Q / W$ 's, the total number of nuclei $Q$ produced by an accelerated particle distribution normalized to the integral cosmic-ray energy $W$, for a given source energy spectrum, and cosmic ray and ambient medium compositions as described above. We make the reasonable assumption that the accelerated particle source energy spectrum is independent of $[\mathrm{Fe} / \mathrm{H}]$. The resultant CRS and CRI $Q / W$ 's for Be and ${ }^{6} \mathrm{Li}$ (left panel) are for $\mathrm{X}_{\mathrm{esc}}=10 \mathrm{gcm}^{-2}$, typical of currently inferred values for "leaky box" cosmic-ray propagation models. While $\alpha \alpha$ dominated $Q\left({ }^{6} \mathrm{Li}\right) / W$ is not very different for the CRS and CRI models, $Q(\mathrm{Be}) / W$ is drastically different for the two models, reflecting the fact that efficient Be production in the early Galaxy can only result from $\mathrm{C}$ and $\mathrm{O}$ enriched accelerated particles. The different $\mathrm{O}$ abundances employed in the calculation of $Q / W$ for the two mixing delays cases (see Figure 2) lead to significantly different $Q(\mathrm{Be}) / W$ 's for CRI model. The LECR $Q / W$ 's (right panel) show that, while removal of the protons and $\alpha$ particles (the CRS(metal) composition) significantly increases $Q / W$ for Be, it essentially leaves $Q\left({ }^{6} \mathrm{Li}\right) / W$ unchanged, because the lack of ${ }^{6} \mathrm{Li}$ production by $\alpha$ particles is compensated by a smaller $\mathrm{W}$ due to the absence of the protons and alphas.

Figure 4 shows the Be evolution for the CRS and CRI models. In the calculations, $10^{50}$ erg per supernova are imparted to the cosmic rays, a value in very good agreement with current epoch cosmic-ray energetics. We note that even though the overall slope of $\log (\mathrm{Be} / \mathrm{H})$ vs. $[\mathrm{Fe} / \mathrm{H}]$ is practically unity, while 

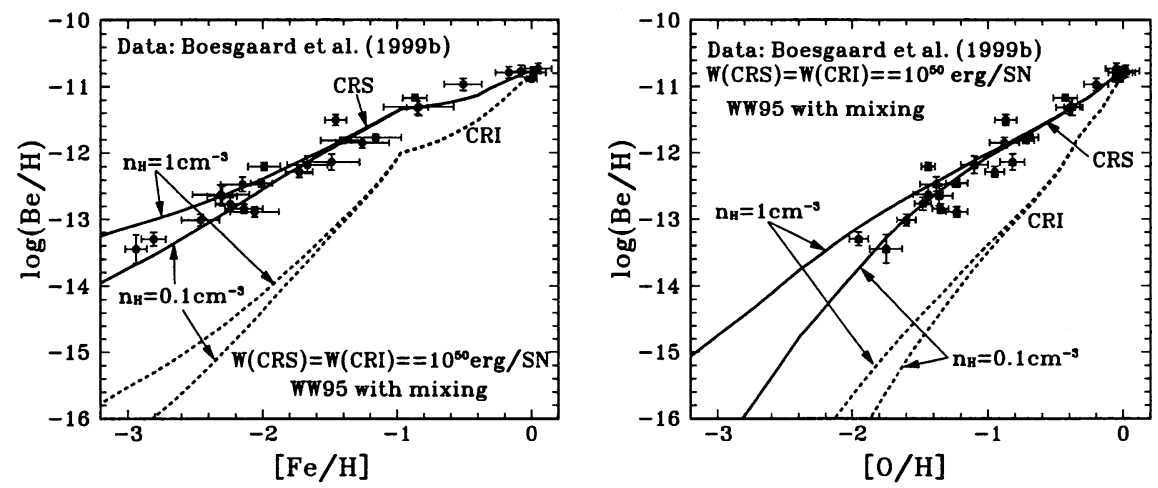

Figure 4. Be abundance evolution for the CRS and CRI models, as a function of $[\mathrm{Fe} / \mathrm{H}]$ (left panel) and $[\mathrm{O} / \mathrm{H}]$ (right panel). $\mathbf{n}_{\mathrm{H}}$ is the density of ambient hydrogen which influences the propagation of the cosmic rays and determines the delay between the supernova explosion and the deposition of the synthesized Be. For both the CRS and CRI model, $10^{50}$ ergs per supernova are imparted to the cosmic rays. Results for only WW95 with mixing delays are shown.

that of $\log (\mathrm{Be} / \mathrm{H})$ vs. $[\mathrm{O} / \mathrm{H}]$ is significantly steeper $(0.96 \pm 0.04$ and $1.45 \pm 0.04$, respectively, Boesgaard et al. 1999b), the CRS model provides a good fit to these evolutionary trends, particularly if $n_{H}$ is near 0.1 . Such a low value might not be unreasonable for an average halo hydrogen density if $10^{10} \mathrm{M}_{\odot}$ are spread over a few $\mathrm{kpc}^{3}$. The calculated $\log (\mathrm{Be} / \mathrm{H})$ vs. $[\mathrm{Fe} / \mathrm{H}]$ is flatter than $\log (\mathrm{Be} / \mathrm{H})$ vs. $[\mathrm{O} / \mathrm{H}]$ because the delayed deposition of the synthesized $\mathrm{Be}$, caused by the low $\mathbf{n}_{\mathrm{H}}$, is compensated by the delayed $\mathrm{Fe}$ deposition, due to the incorporation of $\mathrm{Fe}$ in high velocity dust, but not compensated by the very short delay of the deposition of $\mathrm{O}$, which is mostly volatile. As in Ramaty et al. (2000), we see that the CRI model, normalized to a reasonable energy in cosmic rays per supernova, severely underproduces the measured $\mathrm{Be}$ abundances. However, unlike in that paper where we showed the result only for $\log (\mathrm{Be} / \mathrm{H})$ vs. $[\mathrm{Fe} / \mathrm{H}]$, here we show that the same result also holds for $\log (\mathrm{Be} / \mathrm{H})$ vs. $[\mathrm{O} / \mathrm{H}]$. This removes the remaining ambiguity concerning our argument against the result of Fields \& Olive (1999), who claimed that the CRI model would be viable if instead of $\mathrm{Fe}$ ejecta per supernova based on calculations, which are somewhat uncertain, they used values based on their fit to the increasing $[\mathrm{O} / \mathrm{Fe}]$ with decreasing $[\mathrm{Fe} / \mathrm{H}]$. As there is no such uncertainty concerning the $\mathrm{O}$ ejected masses, our present result unequivocally demonstrates that the CRI model is untenable.

Figure 5 shows the Be evolution for the CRI+LECR model. Here, as before, $10^{50}$ erg per supernova are imparted to the CRI cosmic rays, but in order to achieve a fit to the $\log (\mathrm{Be} / \mathrm{H})$ vs. $[\mathrm{Fe} / \mathrm{H}]$ data, we had to add more energy $\left(1.5 \times 10^{50}\right.$ erg per supernova) in LECRs. The need for this increased energy can be seen in Figure 3, where the $Q(\mathrm{Be}) / W$ for the LECR model with the CRS composition (right panel) is lower by about a factor of 2 than the corresponding value for the CRS model (left panel). Returning to Figure 5, we see that the CRI+LECR model leads to a $\log (\mathrm{Be} / \mathrm{H})$ vs. $[\mathrm{O} / \mathrm{H}]$ evolutionary curve with a 

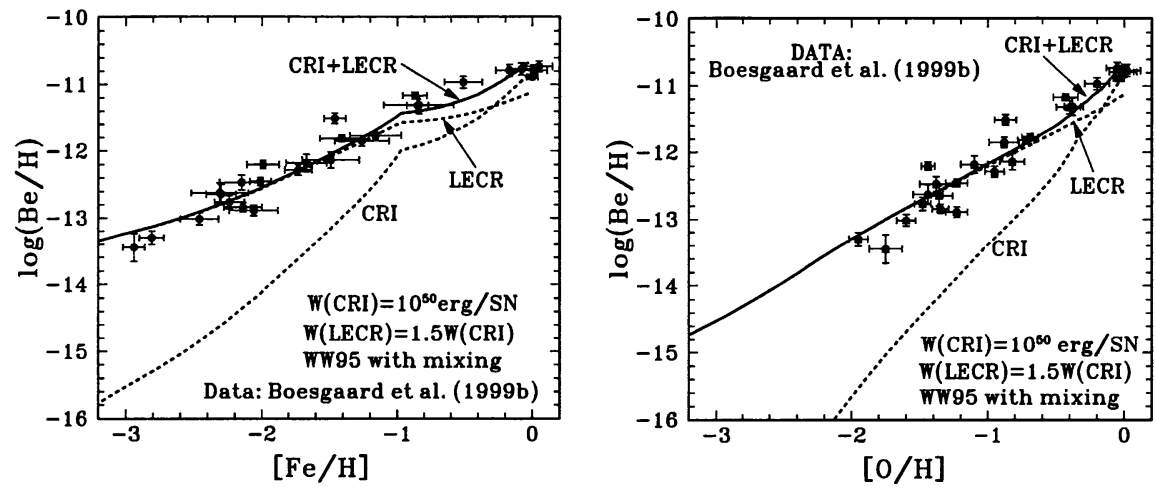

Figure 5. Be abundance evolution for the CRI+LECR model with a turnover energy of $30 \mathrm{MeV} /$ nucleon, as a function of [Fe/H] (left panel) and $[\mathrm{O} / \mathrm{H}]$ (right panel). LECRs slow down much faster than higher energy cosmic rays, hence there is no delay between the supernova explosion and the deposition of the Be. $10^{50}$ ergs per supernova are imparted to the CRI cosmic rays and $50 \%$ more to the LECRs.

slope which is only slightly steeper than 1 , while, as mentioned above, the data indicate a slope of $1.45 \pm 0.04$ (Boesgaard et al. 1999b). Indeed, the simplest evolutionary considerations for both the CRS and LECR models would predict that $\log (\mathrm{Be} / \mathrm{H})$ vs. $[\mathrm{O} / \mathrm{H}]$ should have a slope of 1 . But, as shown above, the delay introduced by cosmic-ray transport with $\mathrm{n}_{\mathrm{H}}=0.1$ will steepen the slope. However, the LECRs slow down much faster than the higher energy CRS cosmic rays. Thus, the calculation of Figure 5 assigns no delay to the Be deposition, leading to a possible inconsistency between the predictions of the CRI+LECR model and the $\log (\mathrm{Be} / \mathrm{H})$ vs. $[\mathrm{O} / \mathrm{H}]$ data.

Figure 6 shows the boron isotopic ratio (left panel) and $\log (\mathrm{B} / \mathrm{Be})$ (right panel) vs. $\mathrm{Fe} / \mathrm{H}$ for the $\mathrm{CRS}$ and $\mathrm{CRI}+\mathrm{LECR}$ models. In the evolutionary calculations, the production ratios $Q\left({ }^{11} \mathrm{~B} /{ }^{10} \mathrm{~B}\right)=2.4$ and $Q(\mathrm{~B} / \mathrm{Be})=14$ for the CRS and CRI cosmic rays, and $Q\left({ }^{11} \mathrm{~B} /{ }^{10} \mathrm{~B}\right)=3.3$ and $Q(\mathrm{~B} / \mathrm{Be})=22$ for the LECRs, were taken as independent of $[\mathrm{Fe} / \mathrm{H}]$. These numerical values are from Ramaty et al. (1997) and are valid for high energy CRS and CRI cosmic rays and LECRs with $E_{0}=30 \mathrm{MeV} /$ nucleon. In order to reproduce the meteoritic ${ }^{11} \mathrm{~B} /{ }^{10} \mathrm{~B}$ both the CRS and CRI+LECR models require the addition of $\nu$-produced ${ }^{11} \mathrm{~B}$ (Woosley \& Weaver 1995). We take into account the metallicity dependence of this ${ }^{11} \mathrm{~B}$ production, and the fact that only the core collapse supernovae produce ${ }^{11} \mathrm{~B}$ by neutrinos. We find that for the CRS model the meteoritic data can be fit with $f_{\nu}=0.18$, a lower value than we found in Ramaty et al. (2000) because of the lower cosmic-ray energy per supernova that we use here $\left(10^{50}\right.$ vs. $1.5 \times 10^{50}$ erg that we used in that paper). The rise in ${ }^{11} \mathrm{~B} /{ }^{10} \mathrm{~B}$ and $\mathrm{B} / \mathrm{Be}$ for $\mathrm{n}_{\mathrm{H}}=0.1$ below $[\mathrm{Fe} / \mathrm{H}]$ of about -2 in the CRS model is due to the delayed deposition of the cosmic-ray produced $\mathrm{Be}$ and $\mathrm{B}$ relative to the $\nu$-produced ${ }^{11} \mathrm{~B}$ for which we took a short delay time, the same as for O $(1 \mathrm{Myr})$. For the CRI+LECR model, in which the LECRs employ a larger energy per supernova, $f_{\nu}=0.2$ is required to fit the boron isotope data. The rise in ${ }^{11} \mathrm{~B} /{ }^{10} \mathrm{~B}$ and $\mathrm{B} / \mathrm{Be}$ with decreasing 

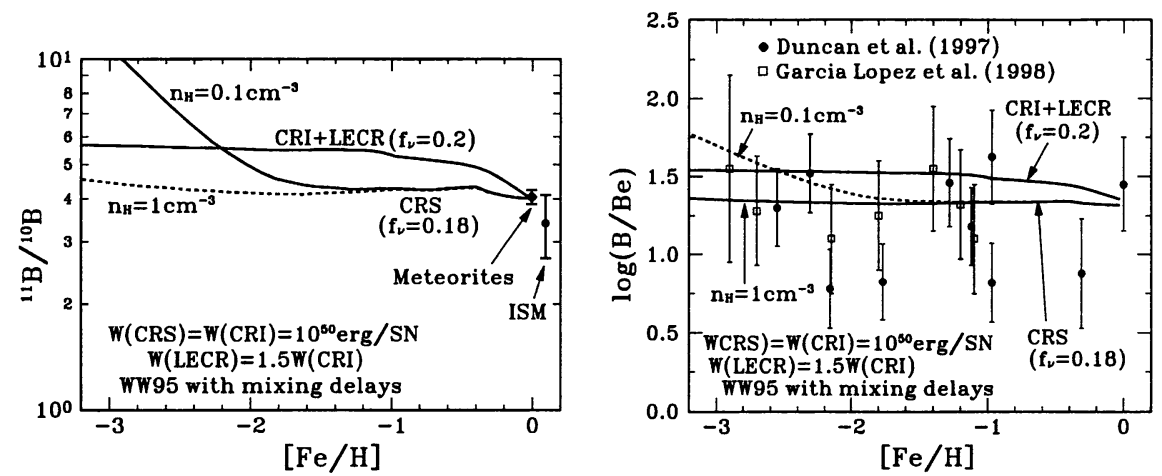

Figure 6. ${ }^{11} \mathrm{~B}-$ to- $^{10} \mathrm{~B}$ (left panel) and B-to-Be (right panel) abundance ratios vs. $[\mathrm{Fe} / \mathrm{H}]$ for the CRS and CRI+LECR models. $f_{\nu}$ is the ratio of the employed $\nu$-produced ${ }^{11} \mathrm{~B}$ to the nominal Woosley \& Weaver (1995) yields. $\mathrm{n}_{\mathrm{H}}$ is defined in the caption of Figure 4. The B isotope data for meteorites and the ISM are from Chaussidon \& Robert (1995) and Lambert et al. (1998), respectively.

$[\mathrm{Fe} / \mathrm{H}]$ is mostly due to the larger value of the corresponding LECR production ratios. It is evident from Figure 6 that future high precision measurements of ${ }^{11} \mathrm{~B} /{ }^{10} \mathrm{~B}$ and $\mathrm{B} / \mathrm{Be}$ as functions of $[\mathrm{Fe} / \mathrm{H}]$ will distinguish between the models.

Figure 7 shows the $\mathrm{Li}$ evolution. The CRS model underproduces the ${ }^{6} \mathrm{Li}$ abundance for $[\mathrm{Fe} / \mathrm{H}]<-2$, suggesting the existence of pregalactic or extragalactic ${ }^{6} \mathrm{Li}$ sources. With the lower cosmic-ray normalization mentioned above, the CRS model also slightly underproduces the meteoritic ${ }^{6} \mathrm{Li}$. With the discovery of solar flare produced ${ }^{6} \mathrm{Li}$ in the solar wind (via measurements in lunar soil, Chaussidon \& Robert 1999), the possibility of some locally produced ${ }^{6} \mathrm{Li}$ in the solar system must be considered. The CRI+LECR model produces more ${ }^{6} \mathrm{Li}$ relative to $\mathrm{Be}$, simply because the $\alpha \alpha$ cross section for ${ }^{6} \mathrm{Li}$ production peaks in the nonrelativistic region.

\section{Discussion and Conclusions}

We have summarized a complete set of $\mathrm{O}, \mathrm{Fe}$ and $\mathrm{LiBeB}$ evolutionary calculation. We have considered the three principal evolutionary models, CRI in which the cosmic-ray source composition at all epochs of Galactic evolution is similar to that of the average ISM at that epoch, CRI+LECR in which metal enriched low energy cosmic rays (LECRs) are superimposed onto the CRI cosmic rays, and CRS in which the cosmic-ray source, accelerated in superbubbles, has a constant composition, independent of the ISM metallicity. By considering the evolutionary trend of $\log (\mathrm{Be} / \mathrm{H})$ vs. both $[\mathrm{Fe} / \mathrm{H}]$ and $[\mathrm{O} / \mathrm{H}]$, we demonstrated that the CRI model is energetically untenable. Although the CRI+LECR mix considered here is consistent with the Be evolution vs. Fe, a plausible scenario for producing the required mix has yet to be proposed.

For $\mathrm{Fe}$, our code allows for a delay between nucleosynthesis and deposition into star forming regions due to the incorporation of the synthesized $\mathrm{Fe}$ into 

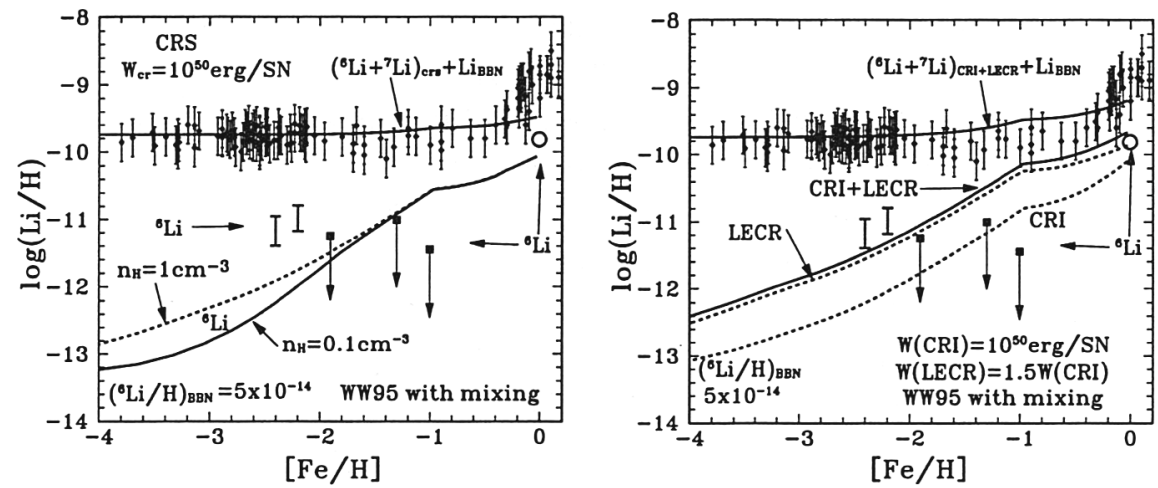

Figure 7. The evolution of the total $\mathrm{Li}$ and ${ }^{6} \mathrm{Li}$ for the CRS (left panel) and the CRI+LECR models (right panel). The ${ }^{6} \mathrm{Li}$ data at $[\mathrm{Fe} / \mathrm{H}]$ of $-2.4,-2.2,-1.9,-1.3$ and -1 are from the summary of Hobbs (1999), and the meteoritic value at $[\mathrm{Fe} / \mathrm{H}]=0$ is from Grevesse et al. (1996). The total $\mathrm{Li}$ data is from a compilation by M. Lemoine (private communication 1997). $\mathrm{n}_{\mathrm{H}}$ is defined in the caption of Figure 4. We took the primordial ${ }^{7} \mathrm{Li} / \mathrm{H}=1.8 \times 10^{-10}$ (Molaro 1999).

high velocity dust. This delay could provide an explanation for the possible rise of $[\mathrm{O} / \mathrm{Fe}]$ with decreasing $[\mathrm{Fe} / \mathrm{H}]$ indicated by some of the data. A test for this scenario would be the demonstration that the abundances of refractory $\alpha$ elements $\mathrm{Mg}, \mathrm{Si}, \mathrm{Ca}$ and $\mathrm{Ti}$ relative to $\mathrm{Fe}$ do not increase with decreasing $[\mathrm{Fe} / \mathrm{H}]$ below $[\mathrm{Fe} / \mathrm{H}]=-1$, but that volatile sulfur does rise.

For the $\mathrm{LiBeB}$ there is also a delay. Due to the transport of the cosmic rays, $\mathrm{LiBeB}$ synthesis lags behind the explosion of the supernova responsible for accelerating the cosmic rays by as much a hundred million years, depending on the average gas density in the halo of the early Galaxy. We show that this delay, combined with the delayed $\mathrm{Fe}$ deposition, could provide an explanation for the steeper evolutionary trend $\log (\mathrm{Be} / \mathrm{H})$ vs. $[\mathrm{O} / \mathrm{H}]$ than vs. $[\mathrm{Fe} / \mathrm{H}]$, as indicated by the recent data of Boesgaard et al. (1999b).

The trends of ${ }^{11} \mathrm{~B} /{ }^{10} \mathrm{~B}$ and $\mathrm{B} / \mathrm{Be}$ vs. $[\mathrm{Fe} / \mathrm{H}]$ show structure resulting from the above mentioned delays, as well as from the hybrid nature of the CRI+LECR model. Future observations of these ratios may distinguish between the models. The fact that the ${ }^{6} \mathrm{Li}$ abundances at $[\mathrm{Fe} / \mathrm{H}]$ below -2 seem inconsistent with all the models suggests the existence of pregalactic or extragalactic ${ }^{6} \mathrm{Li}$ sources.

\section{References}

Bloemen, H. et al. 1994, A\&A, 281, L5

Bloemen, H. et al. 1999, ApJ, 521, L137

Boesgaard, A., King, J., Deliyannis, C., \& Vogt, S. 1999a, AJ, 117, 492

Boesgaard, A., Deliyannis, C., King, J., Ryan, S., Vogt, S., \& Beers, T. 1999b, AJ, 117, 1548 
Bykov, A., \& Bloemen, H. 1994, A\&A, 283, L1

Bykov, A. M., \& Fleishman, G. D. 1992, MNRAS, 15, 269

Cassé, M., Lehoucq, R., \& Vangioni-Flam, E. 1995, Nature, 373, 318

Chaussidon, M., \& Robert, F. 1995, Nature, 374, 337

Chaussidon, M., \& Robert, F. 1999, Nature, 402, 270

Duncan, D. K., Lambert, D. L., \& Lemke, M., 1992, ApJ, 401, 584

Duncan, D. K. et al. 1997, ApJ, 488, 338

Ellison, D. C., Drury, L.O'C., \& Meyer, J-P. 1997, ApJ, 487, 197

Fields, B. D., \& Olive, K. A. 1999, ApJ, 516, 797

Fulbright, J. P, \& Kraft, R. P. 1999, AJ, 118, 527

Garcia Lopez, R. J. et al. 1998, ApJ, 500, 241

Grevesse, N., Noels, A., \& Sauval, A. J. 1996, in: Cosmic Abundances, eds. S.

S. Holt, \& G. Sonneborn, ASP Conf. Ser., 99, (San Francisco: ASP), 117

Higdon, H. C., Lingenfelter, R. E., \& Ramaty, R., 1998, ApJ, 509, L33

Higdon, H. C., Lingenfelter, R. E., \& Ramaty, R., 1999, 26th Internat. Cosmic

Ray Conf., eds. D. Kieda et al. (Salt Lake City), 4, 144

Hobbs, L. M. 1999, in: LiBeB, Cosmic Rays, and Related X- and Gamma-Rays, eds. R. Ramaty et al., ASP Conf. Ser., 171, (San Francisco: ASP), 23

Israelian, G., Garcia Lopez, R. J., \& Rebolo, R. 1998, ApJ, 507, 805

Lambert, D. L. et al. 1998, ApJ, 494, 614

Lingenfelter, R. E., \& Ramaty, R., 1999, 26th Internat. Cosmic Ray Conf., eds. D. Kieda et al. (Salt Lake City), 4, 148

Lingenfelter, R. E., Ramaty, R., \& Kozlovsky, B. 1998, ApJ, 500, L153

Meyer, J-P., Drury, L. O'C., \& Ellison, D. C. 1997, ApJ, 487, 182

Molaro, P. 1999, in: LiBeB, Cosmic Rays, and Related X- and Gamma-Rays, eds. R. Ramaty et al. , ASP Conf. Ser., 171, (San Francisco: ASP), 6

Parizot, E. M. G., Cassé, M., \& Vangioni-Flam, E. 1997, A\&A, 328,107

Ramaty, R. 1996, A\&A(Suppl.), 120, C373

Ramaty, R., Kozlovsky, B., \& Lingenfelter, R. E. 1996, ApJ, 456, 525

Ramaty, R., Kozlovsky, B., Lingenfelter, R., \& Reeves, H. 1997, ApJ, 488, 730

Ramaty, R., Scully, S., Lingenfelter, R., \& Kozlovsky, B. 2000, ApJ, in press

Reeves, H., Fowler, W. A., \& Hoyle, F. 1970, Nature, 226, 727

Ryan, S. G., Norris, J. E., \& Beers, T. C. 1996, ApJ, 471, 254

Ryan, S., Bessell, M., Sutherland, R., \& Norris, J. 1990, ApJ, 348, L57

Timmes, F. X., Woosley, S. E., \& Weaver, T. A., 1995. ApJ(suppl), 98, 617

Tsujimoto, T., \& Shigeyama, T. 1998, ApJ, 508, L151

Vangioni-Flam E., Cassé, M., Audouze, J., \& Oberto, Y. 1990, ApJ, 364, 568

Vangioni-Flam, E., Cassé, M., Fields, B., \& Olive, K. 1996, A\&A, 468, 199

Westin, J. et al. 1999, ApJ, in press (astro-ph/9910376)

Wiedenbeck, M. E. et al. 1999, ApJ(Letters), 523, L61

Woosley, S. E. \& Weaver, T. A. 1995, ApJS, 101, 181 